\title{
Proton-temperature-anisotropy-driven magnetic fields in plasmas with cold and relativistically hot electrons
}

\author{
N IT IN SH UKLA ${ }^{1} \dagger$ and P. K. SHUKL A ${ }^{1,2} \ddagger$ \\ ${ }^{1}$ Institut für Theoretische Physik IV, Fakultät für Physik und Astronomie, \\ Ruhr-Universität Bochum, D-44780 Bochum, Germany \\ (nitshukla@yahoo.com,ps@tp4.rub.de) \\ ${ }^{2}$ School of Physics, University of KwaZulu-Natal, Durban 4000, South Africa
}

(Received 25 June 2009 and accepted 28 August 2009, first published online 8 October 2009)

\begin{abstract}
We present a dispersion relation for a plane-polarized electromagnetic wave in plasmas composed of cold electrons, relativistically hot electrons and biMaxwellian protons. It is shown that the free energy in proton-temperature anisotropy drives purely growing electromagnetic modes in our three-component plasma. Expressions for the growth rates and thresholds of instabilities are presented. The present results are relevant for explaining the origin of spontaneously generated magnetic fields in laboratory and astrophysical plasmas.
\end{abstract}

\section{Introduction}

About 40 years ago, Weibel [1] discovered a purely growing electromagnetic instability in an unmagnetized plasma with a bi-Maxwellian electron velocity distribution. Thus, the free energy in the electron temperature anisotropy generates quasi-stationary magnetic fields [1, 2]. The thermal Weibel instability may account for spontaneously generated magnetic fields in inertial confinement fusion plasmas [3-8], as well as in interplanetary spaces [9-11] and in astrophysical environments (e.g. cluster of galaxies [12]). The importance of relativistic thermal Weibel instability $[13,14]$ has also been recognized in the context of large-scale magnetic fields in a number of astrophysical sources, such as the gamma-ray bursts and relativistic

$\dagger$ Permanently at the Department of Physics, Umeå University, SE-90187 Umeå, Sweden; also affiliated with Grupo de Lasers e Plasmas/Instituto de Plasmas Fusão Nuclear, Instituto Superior Técnico, Universidade Técnica de Lisboa, 1049-001 Lisboa, Portugal.

$\mp$ Also at the Nonlinear Physics Centre and Centre for Plasma Science and Astrophysics, Ruhr-Universität Bochum, D-44780 Bochum, Germany; Department of Physics, Umeå University, SE-90187 Umeå, Sweden; Max-Planck-Institut für extraterrestrische Physik, D-85741 Garching, Germany; Grupo de Lasers e Plasmas/Instituto de Plasmas Fusao Nuclear, Departamento de Física do Instituto Superior Técnico, Universidade Técnica de Lisboa, 1049-001 Lisboa, Portugal; STFC Centre for Fundamental Physics, Rutherford Appleton Laboratory, Chilton, Dideot, Oxon 0X11 0QX, UK; Scottish Universities Physics Alliance (SUPA), Department of Physics, University of Strathclyde, Glasgow G 40NG, Scotland, UK. 
jets $[15,16]$. Recently, large-scale toroidal magnetic fields have been observed in the Galactic Centre [17], accompanied with splendid filamentary radio arcs [18].

Recent simulations [19] and laboratory experiments [20] have revealed that the absorption of intense laser pulses into electrons produces relativistically hot electron components within a dense plasma. Our objective here is to show that the preexisting proton-temperature anisotropy can generate quasi-stationary magnetic fields in plasmas with cold and relativistically hot electrons. For this purpose, we present a dispersion relation for a plane-polarized electromagnetic wave in our multi-species plasma. The new dispersion relation admits purely growing instabilities. The growth rates and thresholds of the instabilities are presented.

The propagation of the electromagnetic wave is governed by the Faraday law

$$
\frac{\partial \mathbf{B}}{\partial t}=-c \nabla \times \mathbf{E}
$$

and the Maxwell equation

$$
\nabla \times \mathbf{B}=\frac{4 \pi}{c}\left(\mathbf{J}_{c}+\mathbf{J}_{h}+\mathbf{J}_{i}\right)+\frac{1}{c} \frac{\partial \mathbf{E}}{\partial t},
$$

where $c$ is the speed of light in vacuum; $\mathbf{B}$ and $\mathbf{E}$ are the wave magnetic and electric fields, respectively; and $\mathbf{J}_{c}, \mathbf{J}_{h}$ and $\mathbf{J}_{i}$ are the current densities of cold electrons, relativistically hot electrons and hot ions, respectively. For the electromagnetic fields, we express $\mathbf{B}=\nabla \times \mathbf{A}$ and $\mathbf{E}=-c^{-1} \partial \mathbf{A} / \partial t$, where $\mathbf{A}$ is the vector potential. The Coulomb gauge is $\nabla \cdot \mathbf{A}=0$.

We are interested in obtaining a dispersion relation for a plane-polarized electromagnetic wave (with $\mathbf{A}=\hat{\boldsymbol{x}} A_{x} \exp (-i \omega t+i k z)$, where $\hat{\boldsymbol{x}}$ is a unit vector along the $x$-axis in a Cartesian co-ordinate system and $A_{x}$ is the $x$-component of the vector potential, $\omega$ is the frequency, and $k$ is the wave number along the $z$ axis) in our three-species plasmas. The equilibrium distribution functions of cold electrons, hot electrons and hot protons are a delta function, a relativistic Maxwellian distribution function [21] and a bi-Maxwellian distribution function [22, 23], respectively. The current density associated with cold electrons is

$$
\mathbf{J}_{e}=-e n_{c 0} \mathbf{u}_{c},
$$

where $e$ is the magnitude of the electron charge; $n_{c 0}$ is the unperturbed number density of cold electrons; and the cold electron fluid velocity is

$$
\mathbf{u}_{c}=\hat{\boldsymbol{x}} \frac{e A_{x}}{m_{e} c} .
$$

Here $m_{e}$ is the rest mass of electrons.

Furthermore, for $m_{e} c^{2} \ll T_{h}$, where $T_{h}$ is the hot-electron temperature, the current density associated with relativistically hot electrons is [21]

$$
\mathbf{J}_{h}=-\hat{\boldsymbol{x}} \frac{n_{h 0} e^{2} c A_{x}}{3 T_{h}} .
$$

The proton current density $\mathbf{J}_{i}$ in the presence of an equilibrium bi-Maxwellian proton distribution function reads [23]

$$
\mathbf{J}_{i}=\hat{\boldsymbol{x}} \frac{Z_{i}^{2} n_{i 0} e}{m_{i} c^{2}}\left[1+\frac{T_{i \perp}}{T_{i \|}} W(\xi)\right] A_{x},
$$


where $Z_{i}$ is the proton charge state; $n_{i 0}$ is the unperturbed proton number density; $Z_{i} n_{i 0}=n_{c 0}+n_{h 0} ; m_{i}$ is the ion mass; $T_{i \perp}$ and $T_{i \|}$ are the proton temperatures across and along the propagation vector $\boldsymbol{k}=\hat{\boldsymbol{z}} k$, where $\hat{\boldsymbol{z}}$ is the unit vector along the $z$-axis. Furthermore, we have denoted $W(\xi)=-1-\xi Z(\xi)$, where $Z(\xi)$ is the plasma dispersion function [24], with $\xi=\omega / k V_{T \|}$ and $V_{T \|}=\left(2 T_{i \|} / m_{i}\right)^{1 / 2}$.

By using the definition of the electromagnetic fields $\mathbf{B}$ and $\mathbf{E}$, we can combine (1) and (2) and Fourier transform the resultant equations to obtain the dispersion relation

$$
\frac{k^{2} c^{2}}{\omega^{2}}=1-\frac{\omega_{p c}^{2}}{\omega^{2}}-\frac{m_{e} c^{2}}{3 T_{h}} \frac{\omega_{p h}^{2}}{\omega^{2}}-\frac{\omega_{p i}^{2}}{\omega^{2}}\left[1+\frac{T_{i \perp}}{T_{i \|}} W(\xi)\right],
$$

to obtain which we have used (3), (5) and (6). We have denoted $\omega_{p \alpha}=\left(4 \pi n_{\alpha 0} e^{2} /\right.$ $\left.m_{e}\right)^{1 / 2}$ and $\omega_{p i}=\left(4 \pi n_{i 0} Z_{i}^{2} e^{2} / m_{i}\right)^{1 / 2}$, where the subscript $\alpha$ equals $c$ for cold electrons and $h$ for relativistically hot electrons.

We analyze (7) in two limiting cases. First, consider the limit $\xi \gg 1$, so that $G(\xi)=$ $1 / 2 \xi^{2}$. Here we have from $(7)$

$$
\frac{k^{2} c^{2}}{\omega^{2}}=1-\frac{\omega_{p c}^{2}}{\omega^{2}}-\frac{m_{e} c^{2}}{3 T_{h}} \frac{\omega_{p h}^{2}}{\omega^{2}}-\frac{\omega_{p i}^{2}}{\omega^{2}}\left(1+\frac{k^{2} T_{i \perp}}{m_{i} \omega^{2}}\right),
$$

which for $\omega \ll k c$ yields

$$
k^{2} c^{2}+\Omega_{p}^{2}=-\frac{\omega_{p i}^{2}}{\omega^{2}} \frac{k^{2} T_{i \perp}}{m_{i}},
$$

where $\Omega_{p}=\left(\omega_{p c}^{2}+\omega_{p i}^{2}+m_{e} c^{2} \omega_{p h}^{2} / 3 T_{h}\right)^{1 / 2}$. Equation (9) admits a purely growing mode $(\omega=i \gamma)$, with the growth rate

$$
\gamma=\frac{k V_{T \perp} \omega_{p i}}{\sqrt{2}\left(k^{2} c^{2}+\Omega_{p}^{2}\right)^{1 / 2}} .
$$

The threshold is

$$
\frac{T_{i \perp}}{T_{i \|}} \gg \frac{\left(k^{2} c^{2}+\Omega_{p}^{2}\right)}{\omega_{p i}^{2}} .
$$

Second, consider the limit $|\xi| \ll 1$, so that $W(\xi)=-1-i \sqrt{\pi} \xi$. Here (8) with $\omega \ll k c$ yields

$$
1+\frac{\omega_{p i}^{2}}{\left(k^{2} c^{2}+\Omega_{p}^{2}\right)}\left[1-\frac{T_{i \perp}}{T_{i \|}}\left(1+i \sqrt{\pi} \frac{\omega}{k V_{T \|}}\right)\right]=0,
$$

which admits a purely growing solution, with the growth rate

$$
\gamma=\frac{k V_{T \|}}{\sqrt{\pi}} \frac{T_{i \|}}{T_{i \perp}}\left[\frac{T_{i \perp}}{T_{i \|}}-1-\frac{\left(k^{2} c^{2}+\Omega_{p}^{2}\right)}{\omega_{p i}^{2}}\right],
$$

provided that

$$
\frac{T_{i \perp}}{T_{i \|}}>1+\frac{\left(k^{2} c^{2}+\Omega_{p}^{2}\right)}{\omega_{p i}^{2}} .
$$

In summary, we have shown the existence of purely growing electromagnetic instabilities in a plasma with cold electrons, relativistically hot electrons and nonrelativistic bi-Maxwellian protons. It is found that the free energy in protontemperature anisotropy drives purely growing magnetic fields. Proton-anisotropydriven instabilities may saturate when the gyrofrequency in the saturated magnetic 
field $B_{s}$ is comparable to the growth rate of instabilities. The saturated magnetic field can be associated with a large-scale magnetic field which may coexist with cold electrons, relativistically hot electrons and protons having a bi-Maxwellian distribution function. Such a scenario might occur in intense laser-plasma interaction experiments [8] and in astrophysical environments [22]. Finally, we note that the present investigation can be readily generalized for multi-component plasmas with a relativistic bi-Maxwellian proton distribution function. Here we should follow the analysis of Yoon [14] and Mart'yanov et al. [25] for investigating relativistic proton-anisotropy-driven magnetic fields in plasmas.

\section{Acknowledgements}

This research was partially supported by the Deutsche Forschungsgemeinschaft (Bonn) through project SH21/3-1 of Research Unit 1048.

\section{References}

[1] Weibel, E. S. 1959 Phys. Rev. Lett. 2, 83.

[2] Fried, B. D. 1959 Phys. Fluids 2, 337.

[3] Bychenkov, V. Yu., Silin,V. P. and Tikhonchuk, V. T. 1989 Sov. J. Plasma Phys. 15, 407; 1989 Phys. Lett. A 138, 127.

[4] Basu, B. 2002 Phys. Plasmas 9, 5131.

[5] Estabrook, K. 1978 Phys. Rev. Lett. 41, 1808; Honda, M. 2004 Phys. Rev. E 69, 06 401; Romanov, D. V., Bychenkov, V. Yu., Rozmus, W., Capjack, C. E. and Fedosejers, R. 2004 Phys. Rev. Lett. 93, 215004.

[6] Tatarakis, M. et al. 2002 Nature (London) 415, 280; Wagner, U. et al. 2004 Phys. Rev. E 70, 026401 .

[7] Silva, L. O. et al. 2002 Phys. Plasmas 9, 2458; Tschentscher, T. 2001 Proc. SPIE Int. Soc. Opt. Eng. 4500, 1; Eliezer, S., Mendonça, J. T., Bingham, R. and Norreys, P. 2005 Phys. Lett. A 336, 390; Marklund, M. and Shukla, P. K. 2006 Rev. Mod. Phys. 78, 591.

[8] Nilson, P. M., Willingale, L., Kaluza, M. C. et al. 2006 Phys. Rev. Lett. 97, 255001.

[9] Medvedev, M. V. and Loeb, A. 1999 Astrophys. J. 526, 697; Gruzinov, A. 2001 Astrophys. J. 563, L15.

[10] Schlickeiser, R. and Shukla, P. K. 2003 Astrophys. J. 563, L57; Medvedev, M. V., Silva, L. O. and Kamionkowaski, M. 2006 Astrphys. J. 642, L1.

[11] Widrow, L. M. 2002 Rev. Mod. Phys. 74, 775; Kronberg, P. P. 2002 Phys. Today 55, 40; 2003 Phys. Plasmas 10, 1985.

[12] Carilli, C. L. and Taylor, G. B. 2002 Annu. Rev. Astron. Astrophys. 40, 319.

[13] Yoon, P. H. and Davidson, R. C. 1987 Phys. Rev. A 35, 2718; Schlickeiser, R. 2004 Phys. Plasmas 11, 5532.

[14] Yoon, P. H. 2007 Phys. Plasmas 14, 024504.

[15] Fonseca, R. A., Silva, L. O., Tange, J. W. et al. 2003 Phys. Plasmas 10, 1979; Silva, L. O., Fonseca, R. A., Tange, J. W. et al. 2003 Astrophys. J. 596, L121; Fiore, M., Silva, L. O., Ren, C. et al. 2006 Mon. Not. R. Astron. Soc. 372, 1851.

[16] Honda, M. and Honda, Y. S. 2004 Astrophys. J. 617, L37.

[17] Novak, G., Chuss, D. T., Renbarger, T. et al. 2003 Astrophys. J. 583, L83.

[18] Yusuf-Zadeh, F., Morris, M. and Chance, D. 1984 Nature (London) 337, 310; Yusuf-Zadeh, F. and Morris, M. 1987 Astrophys. J. 322, 721.

[19] Ren, C., Tzoufras, M., Tsung, F. S. et al. 2004 Phys. Rev. Lett. 93, 185004. 
[20] Krushelnick, K., Rozmus, W., Wagner, U. et al. 2008 Phys. Rev. Lett. 100, 125005.

[21] Silin, V. P. 1960 Sov. Phys. JETP 11, 1136.

[22] Ren, C., Blackman, E. G. and Fong, W. 2007 Phys. Plasmas 14, 012901.

[23] Shukla, P. K. 2007 Phys. Lett. A 372, 316.

[24] Fried, B. D. and Conte, S. D. 1961 The Plasma Dispersion Function. New York: Academic.

[25] Mart'yanov, V. Yu., Kocharovsky, V. V. and Kocharovsky, VI. V. 2008 JETP 107, 1049. 